\title{
TOPOLOGICAL TRIVIALITY OF FAMILIES OF REAL ISOLATED SINGULARITIES AND THEIR MILNOR FIBRATIONS
}

\author{
RAIMUNDO NONATO ARAÚJO DOS SANTOS*
}

\begin{abstract}
The aim of this paper is to study the topological triviality and the topological equivalence of the Milnor fibrations for families of real analytic map germs with no coalescing of critical points.
\end{abstract}

\section{Introduction}

In the case of complex germs, equivalent conditions to the topological triviality of families of germs of functions with isolated singularity, had been gotten by several authors (see [12], [2], [4]). A theorem due to G. M. Greuel summarizes various of these results, presenting equivalent conditions to the topological triviality of such families. As a consequence of his result it follows that no coalescing of critical points is a necessary and sufficient condition for topological triviality of complex analytic families of function-germs. A natural question is whether or not no coalescing of singularities also is a sufficient condition for topological triviality of families of real analytic function-germs. The answer is not. H. King [5] presents an example showing that the above question is false, and gives sufficient conditions so that a family with no coalescing of critical points is topologically trivial.

The aim of this paper is to introduce sufficient conditions for the topological triviality of families of real analytic map-germs $F: \mathrm{R}^{n} \times \mathrm{R}, 0 \rightarrow \mathrm{R}^{2}$, 0 , with isolated singularities at the origin. The main result is the following:

Theorem 3.1. Let $F(x, t)=(P(x, t), Q(x, t))$ where $P(x, t)=f(x)+$ $t \theta(x), Q(x, t)=g(x)+t \alpha(x)$ be a family of analytic map-germs. Suppose that the following conditions hold:

(A)

$$
\frac{\left|\left\langle\partial P_{t}(x), \partial Q_{t}(x)\right\rangle\right|}{\left\|\partial P_{t}(x)\right\|\left\|\partial Q_{t}(x)\right\|} \leq 1-\rho,
$$

\footnotetext{
* Research partially supported by ICMC/USP, São Paulo, Brazil.

Received July 30, 2003.
} 
in the neighborhood $V$ of 0 in $\mathbf{R}^{n}$, for all $t \in \mathbf{R}$ and $0<\rho \leq 1$. $\left(B_{f}\right)$

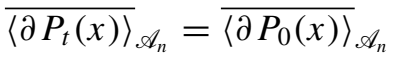

$$
\begin{aligned}
& {\overline{\left\langle\partial Q_{t}(x)\right\rangle_{\mathscr{A}}}}={\overline{\left\langle\partial Q_{0}(x)\right\rangle_{\mathscr{A}}}},
\end{aligned}
$$

for all $t \in \mathbf{R}$.

Then the family $F_{t}$ is topologically trivial.

The conditions $(A)$ and $\left(B_{f}\right)$ are inspired in the conditions introduced by A. Jacquemard ([7, Theorem 1]) to study the Milnor fibrations to real analytic singularities of the kind $f=(P, Q): \mathbf{R}^{n}, 0 \rightarrow \mathbf{R}^{2}, 0$.

\section{Previous results}

Let $F:\left(\mathrm{R}^{p} \times \mathrm{R}^{n}, \mathrm{R}^{p} \times 0\right) \rightarrow\left(\mathrm{R}^{k} \times 0\right)$ be a one-parameter family of map germs with isolated singularity, $F_{t}(x)=F(x, t)$. We will usually denote a family of germs by $F_{t}:\left(\mathrm{R}^{n}, 0\right) \rightarrow\left(\mathrm{R}^{k}, 0\right), t \in \mathrm{R}^{p}$, where $F_{t}(x)=F(t, x)$.

Definition 2.1. A family $F:\left(\mathrm{R}^{p} \times \mathrm{R}^{n}, \mathrm{R}^{p} \times 0\right) \rightarrow\left(\mathrm{R}^{k}, 0\right)$ is said to have no coalescing of critical points (or to be a good deformation of $F_{0}$ ) if there is a neighborhood $U$ of $\mathrm{R}^{p} \times 0$ in $\mathrm{R}^{p} \times \mathrm{R}^{n}$ and a representative $G$ of $F$ so that $G$ restricted to $U \cap\left(t \times\left(\mathrm{R}^{n} \backslash 0\right)\right.$ is a submersion for each $t \in \mathrm{R}^{p}$. Otherwise, the family is said to coalesce.

Definition 2.2. The germs $f_{i}:\left(\mathrm{R}^{n}, 0\right) \rightarrow\left(\mathrm{R}^{k}, 0\right), i=0,1$ are topologically right-equivalent $\left(\mathscr{C}^{0}-\mathscr{R}\right.$-equivalent) if there is a germ of a homeomorphism $h:\left(\mathrm{R}^{n}, 0\right) \rightarrow\left(\mathrm{R}^{n}, 0\right)$ so that the germs $f_{0} h$ and $f_{1}$ are the same. The topological type of a germ is its topological right equivalence class.

Definition 2.3. We say that a family of map-germs $F_{t}:\left(\mathrm{R}^{n}, 0\right) \rightarrow\left(\mathrm{R}^{k}, 0\right)$, $t \in \mathrm{R}^{p}$ and $F_{t}(x)=F(t, x)$, is topologically trivial if there is a continuous family of germs of homeomorphisms $G_{t}:\left(\mathrm{R}^{n}, 0\right) \rightarrow\left(\mathrm{R}^{n}, 0\right), t \in \mathrm{R}^{p}$, such that $F_{0}(x)=F_{t} \circ G_{t}(x)$.

H. King [6] presents an example of a family of real analytic functions which does not have coalescing of critical points and is not topolo-gically trivial; moreover, he gives sufficient conditions so that a good deformation is topologically trivial. To state King's results we need the following definition.

Definition 2.4. For a polynomial $f:\left(\mathrm{R}^{n}, 0\right) \rightarrow\left(\mathrm{R}^{k}, 0\right), n \geq p$, with an isolated critical point at 0 , define $r(f)$, the Milnor radius of $f$, to be the smallest critical value of the distance function $\|x\|^{2}$ restricted to $f^{-1}(0) \backslash 0$ (or $\infty$ if there are no critical values). In other words, $r(f)$ is the biggest $\epsilon_{0}>0$ such that there is $0<\epsilon \leq \epsilon_{0}$ with $\left(f^{-1}(0) \backslash 0\right) \bar{\pitchfork} \boldsymbol{S}_{\epsilon}^{n-1}$. 
In [6], H. King shows the following results:

THeOREM 2.5. Suppose $F_{t}:\left(\mathrm{R}^{n}, 0\right) \longrightarrow\left(\mathrm{R}^{k}, 0\right), t \in \mathrm{R}^{p}$ is a conti-nuous family of polynomial germs with no coalescing of critical points and there is a $\delta>0$ so that $r\left(F_{t}\right)>\delta$ for all $t \in \mathrm{R}^{p}$. Then there is a continuous family of homeomorphism germs $H_{t}:\left(\mathrm{R}^{m}, 0\right) \rightarrow\left(\mathrm{R}^{m}, 0\right)$ so that $F_{0}=F_{t} \circ H_{t}$ for all $t \in \mathrm{R}^{p}$.

THEOREM 2.6. Let $F_{t}:\left(\mathrm{R}^{n}, 0\right) \longrightarrow\left(\mathrm{R}^{k}, 0\right), t \in \mathrm{R}^{p}$ be a family of germs with no coalescing of critical points and suppose there is a family of homeomorphism germs $G_{t}:\left(\mathrm{R}^{m}, 0\right) \longrightarrow\left(\mathrm{R}^{m}, 0\right), t \in \mathrm{R}^{p}$ so that the germ at 0 of each set $G_{t} \circ F_{t}^{-1}(0)$ is the germ of $F_{0}^{-1}(0)$. Then there is a family of homeomorphism germs $H_{t}:\left(\mathrm{R}^{m}, 0\right) \longrightarrow\left(\mathrm{R}^{m}, 0\right), t \in \mathrm{R}^{p}$ and a neighborhood $V$ of 0 in $\mathrm{R}^{p}$ so that the germ at 0 of $F_{t} \circ H_{t}$ is the germ of $F_{0}$ for each $t \in V$.

The $\mathscr{C}^{0}-\mathscr{R}$-equivalence class of $f_{0}$ relates to the equivalence class of its Milnor fibration. More precisely, given $f_{0}, g_{0}:\left(\mathbf{R}^{n}, 0\right) \rightarrow\left(\mathbf{R}^{k}, 0\right)$ with isolated singularity, $f_{0}$ is $\mathscr{C}^{0}-\mathscr{R}$-equivalent to $g_{0}$ if and only if their Milnor fibrations are also equivalent. (See [5, Theorem 1]).

Recall that if $f:\left(\mathrm{C}^{n+1}, 0\right) \rightarrow(\mathrm{C}, 0)$ is the germ of a holomorphic function with a critical point at 0 , then for every sufficiently small $\epsilon>0$, the map $\phi:=\frac{f}{\|f\|}: \mathscr{S}_{\epsilon}^{2 n+1} \backslash \mathscr{S}^{1}$, is the projection map of a locally trivial fiber bundle, where $K=f^{-1}(0) \cap \mathscr{S}_{\epsilon}$ is the link of 0 . This is the Milnor fibration of $f$. Milnor also proves in the last chapter of his book a fibration theorem for real singularities. He shows that if $f:\left(\mathrm{R}^{n}, 0\right) \rightarrow\left(\mathrm{R}^{p}, 0\right), n \geq p \geq 2$, is the germ of a real analytic map-germ whose derivative $D f$ has rank $p$ on a punctured neighborhood of $0 \in \mathrm{R}^{n}$, then, for every sufficiently small sphere $\mathscr{S}_{\epsilon}^{n-1} \subset \mathrm{R}^{n}$ centered at 0 , one has a locally trivial fiber bundle, $f=\frac{f}{\|f\|}: \mathscr{S}_{\epsilon}^{n-1} \backslash N_{K} \rightarrow$ $\mathscr{S}^{p-1}$, where $N_{K}$ denotes a tubular neighborhood of the link $K$ in $\mathscr{S}_{\epsilon}^{n-1}$.

Moreover, $f$ can be extended to $\mathscr{S}_{\epsilon}^{n-1} \backslash K$ as the projection map of a fiber bundle, but this extension may not be given by the obvious map $\frac{f}{\|f\|}$. The problem of studying real isolated singularities at 0 , for which the map $\frac{f}{\|f\|}$ extends to all of $\mathscr{S}^{n-1} \backslash K \rightarrow \mathscr{S}^{p-1}$ as the projection map of a fiber bundle (as in the case of holomorphic maps) was first studied by A. Jacquemard in [7] (see also [8]).

Let $f=(P, Q):\left(\mathrm{R}^{n}, 0\right) \rightarrow\left(\mathrm{R}^{2}, 0\right)$ be a real analytic map-germ with an isolated singularity at 0 . Let $\mathscr{A}_{n}$ be the ring of real analytic germs in $\mathrm{R}^{n}, 0$ and $I=\langle\partial P(x)\rangle$ (resp. $\langle\partial Q(x)\rangle$ ) the ideal in $\mathscr{A}_{n}$ generated by the partial derivatives of $P$ (resp. $Q$ ), and by $\bar{I}$ the integral closure of $I$, that is, $\bar{I}=\{h \in$ $\mathscr{A}_{n} \mid \exists a_{i} \in I^{i}$ with $\left.h^{n}+a_{1} h^{n-1}+\cdots+a_{n}=0\right\}$.

TheOREM ([7, Theorem 1]). Suppose that there exists a neighborhood $V$ 
of 0 in $\mathrm{R}^{n}$ such that:

(A)

$$
\frac{|\langle\partial P(x), \partial Q(x)\rangle|}{\|\partial P(x)\|\|\partial Q(x)\|} \leq 1-\rho,
$$

in the neighborhood $V$ of 0 in $\mathrm{R}^{n}, 0<\rho \leq 1$.

(B)

$$
\overline{\langle\partial P(x)\rangle}=\overline{\langle\partial Q(x)\rangle} .
$$

Then there exists a sufficiently small $\epsilon_{0}>0$, such that for all $0<\epsilon \leq \epsilon_{0}$, $\phi=\frac{f}{\|f\|}: \mathscr{S}_{\epsilon}^{n-1} \backslash K_{\epsilon} \rightarrow \mathscr{S}^{1}$ is the projection map of a locally trivial fiber bundle. Furthermore, this fiber bundle is equivalent to the fibration given Milnor in [9].

In [11] the authors replace the condition $(B)$ in Theorem above by condition

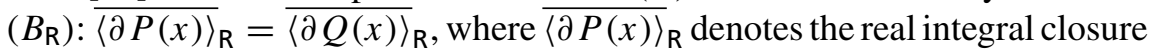
as defined by $\mathrm{T}$. Gaffney [3]. In the complex analytic category, both conditions are equivalent (see [3], [12]).

Definition 2.7. Let $I$ be an ideal in the ring $\mathscr{A}_{n}$. The real integral closure of $I$, denoted by $\overline{I_{\mathrm{R}}}$, is the set of $h \in \mathscr{A}_{n}$ such that for all real analytic curve $\gamma:(\mathrm{R}, 0) \rightarrow\left(\mathrm{R}^{n}, 0\right)$, we have $h \circ \gamma \in\left(\gamma^{*}(I)\right) \mathscr{A}_{1}$.

\section{The Main Theorem}

In this section we present the main result of this paper as well as its main consequences. Let $f=\left(P_{0}, Q_{0}\right):\left(\mathrm{R}^{n}, 0\right) \rightarrow\left(\mathrm{R}^{2}, 0\right)$, be the germ of a real analytic map-germ with isolated singularity at zero, and $F:\left(\mathrm{R}^{n} \times \mathrm{R}, 0\right) \rightarrow$ $\left(\mathrm{R}^{2}, 0\right)$ be a deformation of $f$ given by $F(x, t)=(P(x, t), Q(x, t)), P(x, t)=$ $P_{0}(x)+t \theta(x), Q(x, t)=Q_{0}(x)+t \alpha(x)$.

Theorem 3.1. Let $F$ be as above and $\partial_{x} P(x, t)=\operatorname{Grad}_{x} P_{t}(x), \partial P=$ $\left(\partial_{x} P(x, t), \frac{\partial P}{\partial t}\right)$. Suppose that the following conditions hold:

(A)

$$
\frac{\left|\left\langle\partial_{x} P(x, t), \partial_{x} Q(x, t)\right\rangle\right|}{\left\|\partial_{x} P(x, t)\right\|\left\|\partial_{x} Q(x, t)\right\|} \leq 1-\rho,
$$

$x \neq 0$ near zero, for all $t \in \mathrm{R}$ and $0<\rho \leq 1$.

$\left(B_{f}\right)$

$$
\begin{aligned}
& \overline{\left\langle\partial_{x} P(x, t)\right\rangle_{\mathrm{R}}}=\overline{\left\langle\partial_{x} P(x, 0)\right\rangle_{\mathrm{R}}} \\
& \overline{\left\langle\partial_{x} Q(x, t)\right\rangle_{\mathrm{R}}}=\overline{\left\langle\partial_{x} Q(x, 0)\right\rangle_{\mathrm{R}}},
\end{aligned}
$$

for all $t \in \mathrm{R}$. 
Then the family $F_{t}$ is topologically trivial.

Proof. The idea of the proof is to construct a vector field $V(x, t)=$ $a(x, t) \partial_{x} P(x, t)+b(x, t) \partial_{x} Q(x, t)+\frac{\partial}{\partial t}$ (where $\frac{\partial}{\partial t}$ is the unit vector in $t$ direction) such that:

$$
\left\{\begin{array}{l}
\langle\partial P(x, t), V(x, t)\rangle=0 \\
\langle\partial Q(x, t), V(x, t)\rangle=0
\end{array}\right.
$$

The vector field $V(x, t)$ is tangent to the levels $X=F^{-1}(c)=P^{-1}\left(c_{1}\right) \cap$ $Q^{-1}\left(c_{2}\right)$, where $c=\left(c_{1}, c_{2}\right) \in \mathrm{R}^{2}$. In particular for $c=0, V$ is tangent to the variety $F^{-1}(0)=P^{-1}(0) \cap Q^{-1}(0)$.

The flow $\phi(x, t), \phi(x, 0)=x$, satisfies the following:

$$
\left\{\begin{array}{l}
\frac{\partial P(\phi(x, t))}{\partial t}=\langle\partial P, V\rangle=0 \\
\frac{\partial Q(\phi(x, t))}{\partial t}=\langle\partial Q, V\rangle=0 .
\end{array}\right.
$$

Hence, $F(\phi(x, t))=F(\phi(x, 0))=(f(x), g(x))$.

From (1) it follows that:

$$
\left\{\begin{array}{l}
\langle\partial P, V\rangle=a\left\|\partial_{x} P\right\|^{2}+b\left\langle\partial_{x} P, \partial_{x} Q\right\rangle+\frac{\partial P}{\partial t}=0 \\
\langle\partial Q, V\rangle=a\left\langle\partial_{x} P, \partial_{x} Q\right\rangle+b\left\|\partial_{x} Q\right\|^{2}+\frac{\partial Q}{\partial t}=0
\end{array}\right.
$$

The matrix of the system is

$$
\left(\begin{array}{cc}
\left\|\partial_{x} P\right\|^{2} & \left\langle\partial_{x} P, \partial_{x} Q\right\rangle \\
\left\langle\partial_{x} P, \partial_{x} Q\right\rangle & \left\|\partial_{x} Q\right\|^{2}
\end{array}\right)\left(\begin{array}{l}
a \\
b
\end{array}\right)=-\left(\begin{array}{c}
\theta(x) \\
\alpha(x)
\end{array}\right)
$$

and it follows from condition $(A)$ that its determinant:

$$
\Delta(x, t):=\left\|\partial_{x} P(x, t)\right\|^{2}\left\|\partial_{x} Q(x, t)\right\|^{2}-\left\langle\partial_{x} P(x, t), \partial_{x} Q(x, t)\right\rangle^{2}
$$

satisfies the condition $\Delta(x, t) \neq 0$ for all $x \in V$, in a neighborhood of $0 \in \mathrm{R}^{n}$, $\forall t$. Then we can write:

$$
\left(\begin{array}{l}
a \\
b
\end{array}\right)=-\frac{1}{\Delta}\left(\begin{array}{cc}
\left\|\partial_{x} Q\right\|^{2} & -\left\langle\partial_{x} P, \partial_{x} Q\right\rangle \\
-\left\langle\partial_{x} P, \partial_{x} Q\right\rangle & \left\|\partial_{x} P\right\|^{2}
\end{array}\right)\left(\begin{array}{c}
\theta(x) \\
\alpha(x)
\end{array}\right)
$$


Hence,

$$
\left\{\begin{array}{l}
a=-\frac{1}{\Delta}\left(\left\|\partial_{x} Q\right\|^{2} \theta(x)-\left\langle\partial_{x} P, \partial_{x} Q\right\rangle \alpha(x)\right) \\
b=-\frac{1}{\Delta}\left(\left\|\partial_{x} P\right\|^{2} \alpha(x)-\left\langle\partial_{x} P, \partial_{x} Q\right\rangle \theta(x)\right)
\end{array}\right.
$$

This vector field is well defined and smooth in $U$, where $U \subset \mathbf{R}^{n} \times \mathbf{R} \backslash(\{0\} \times \mathbf{R})$. To show that $V$ is integrable at the points $(0, t)$, it is sufficient to guarantee that there exists a constant $C>0$ such that:

$$
\left\|V(x, t)-\frac{\partial}{\partial t}\right\| \leq C\|x\|,
$$

for $x$ sufficiently close to zero. This will follow from Lemma 3.2 and Proposition 3.3 below.

Lemma 3.2. There exist constants $c_{1}>0$ and $c_{2}>0$ such that:

(a) $|\theta(x)| \leq c_{1}\|x\|\left\|\partial_{x} P\right\|$ and

(b) $|\alpha(x)| \leq c_{2}\|x\|\left\|\partial_{x} Q\right\|$.

Proof. (a) The function $\theta(x)$ is analytic and $\theta(0)=0$, then it follows from the Bochnak-Lojasiewicz inequality [1] that,

$$
|\theta(x)| \leq c_{0}\|x\|\left\|\partial_{x} \theta(x)\right\|
$$

On the other hand,

$|t|\left\|\partial_{x} \theta\right\|-\left\|\partial_{x} f(x)\right\| \leq\left\|\partial_{x} f(x)+t \partial_{x} \theta(x)\right\|=\left\|\partial_{x} P(x, t)\right\| \leq c_{1}\left\|\partial_{x} f(x)\right\|$,

where in the last inequality we use condition $(B)$.

Thus, $|t|\left\|\partial_{x} \theta\right\| \leq\left(1+c_{1}\right)\left\|\partial_{x} f(x)\right\| \forall t$.

In particular taking $t=1$, we have

$$
\left\|\partial_{x} \theta\right\| \leq\left(1+c_{1}\right)\left\|\partial_{x} f(x)\right\| .
$$

Now using again condition $(B)$, we have:

$$
\left\|\partial_{x} \theta\right\| \leq\left(1+c_{1}\right)\left\|\partial_{x} f(x)\right\| \leq c_{2}\left\|\partial_{x} P(x, t)\right\|
$$

for all $t$. Therefore,

$$
\left\|\partial_{x} \theta(x)\right\| \leq c_{2}\left\|\partial_{x} P(x, t)\right\|, \quad \forall t
$$

From (7) and (8) it follows that:

$$
\|\theta(x)\| \leq c_{0}\|x\|\left\|\partial_{x} \theta(x)\right\| \leq c\|x\|\left\|\partial_{x} P(x, t)\right\| .
$$


Part (b) can be proved in the same way.

Proposition 3.3. $\left\|V(x, t)-\frac{\partial}{\partial t}\right\| \leq c\|x\|$.

Proof.

$$
\begin{aligned}
\left\|V(x, t)-\frac{\partial}{\partial t}\right\| & =\left\|a(x, t) \partial_{x} P(x, t)+b(x, t) \partial_{x} Q(x, t)\right\| \\
& \leq\left\|a(x, t) \partial_{x} P(x, t)\right\|+\left\|b(x, t) \partial_{x} Q(x, t)\right\|
\end{aligned}
$$

From (5) we have

$$
\begin{aligned}
\| a(x, t) & \partial_{x} P(x, t) \| \\
& =\left|\frac{1}{\Delta}\left(\left\|\partial_{x} Q\right\|^{2} \theta(x)-\left\langle\partial_{x} P, \partial_{x} Q\right\rangle \alpha(x)\right)\right|\left\|\partial_{x} P(x, t)\right\| \\
& \leq \frac{1}{|\Delta|}\left\|\partial_{x} Q\right\|^{2}|\theta(x)|\left\|\partial_{x} P\right\|+\frac{1}{|\Delta|}\left|\left\langle\partial_{x} P, \partial_{x} Q\right\rangle\right||\alpha(x)|\left\|\partial_{x} P\right\| .
\end{aligned}
$$

Furthermore,

$$
\text { (10) } \begin{aligned}
& \frac{1}{|\Delta|}\left\|\partial_{x} Q\right\|^{2}|\theta(x)|\left\|\partial_{x} P\right\|\left(\frac{\left\|\partial_{x} P\right\|^{2}\left\|\partial_{x} Q\right\|^{2}}{\left\|\partial_{x} P\right\|^{2}\left\|\partial_{x} Q\right\|^{2}}\right) \\
& =\frac{|\theta(x)|}{\left\|\partial_{x} P\right\|}\left(\frac{\left\|\partial_{x} P(x, t)\right\|^{2}\left\|\partial_{x} Q(x, t)\right\|^{2}-\left\langle\partial_{x} P(x, t), \partial_{x} Q(x, t)\right\rangle^{2}}{\left\|\partial_{x} P\right\|^{2}\left\|\partial_{x} Q\right\|^{2}}\right)^{-1} \\
& =\frac{|\theta(x)|}{\left\|\partial_{x} P\right\|}\left(1-\frac{\left\langle\partial_{x} P(x, t), \partial_{x} Q(x, t)\right\rangle^{2}}{\left\|\partial_{x} P\right\|^{2}\left\|\partial_{x} Q\right\|^{2}}\right)^{-1} .
\end{aligned}
$$

From condition $(A)$, there exist $\delta, 0<\delta<1$ such that $\frac{\left|\left\langle\partial_{x} P_{t}(x), \partial_{x} Q_{t}(x)\right\rangle\right|^{2}}{\left|\partial_{x} P_{t}(x)\right|^{2}\left|\partial_{x} Q_{t}(x)\right|^{2}} \leq \delta$, hence

$$
1-\frac{\left|\left\langle\partial P_{t}(x), \partial Q_{t}(x)\right\rangle\right|^{2}}{\left\|\partial P_{t}(x)\right\|^{2}\left\|\partial Q_{t}(x)\right\|^{2}} \geq 1-\delta
$$

From Lemma 3.2(a), it follows that

$$
\frac{|\theta(x)|}{\left\|\partial_{x} P\right\|} \leq c_{1}\|x\| .
$$

Replacing (11) and (12) in (10), we obtain

$$
\frac{1}{|\Delta|}\left\|\partial_{x} Q\right\|^{2}|\theta(x)|\left\|\partial_{x} P\right\| \leq c_{2}\|x\|
$$


Similarly, we have

$$
\frac{1}{|\Delta|}\left|\left\langle\partial_{x} P, \partial_{x} Q\right\rangle\right||\alpha(x)|\left\|\partial_{x} P\right\| \leq c_{3}\|x\| .
$$

Replacing (13) and (14) in (9), we get

$$
\left\|a(x, t) \partial_{x} P(x, t)\right\|<c_{4}\|x\| .
$$

In the same way, we can prove that

$$
\left\|b(x, t) \partial_{x} Q(x, t)\right\|<c_{5}\|x\| .
$$

It is clear that condition $(A)$ in Theorem 3.1 is a sufficient condition for the no coalescing of critical points for the family $F(x, t)$. It is not clear whether $(A)$ implies the constance of the Milnor radius of the family. However, we can replace condition $\left(B_{f}\right)$ by a weaker condition namely, condition $\left(B_{f}\right)$ along the zero sets of $P$ and $Q$, and use King's result (see Theorem 2.6) to get the following:

Proposition 3.4. Let $F$ be as above and $\partial_{x} P(x, t)=\operatorname{Grad}_{x} P_{t}(x), \partial P=$ $\left(\partial_{x} P(x, t), \frac{\partial P}{\partial t}\right)$. Suppose that the following conditions hold:

(A)

$$
\frac{\left|\left\langle\partial_{x} P(x, t), \partial_{x} Q(x, t)\right\rangle\right|}{\left\|\partial_{x} P(x, t)\right\|\left\|\partial_{x} Q(x, t)\right\|} \leq 1-\rho,
$$

$x \neq 0$ near zero, for all $t \in \mathrm{R}$ and $0<\rho \leq 1$.

$\left(B_{f}^{*}\right)$

$$
\begin{aligned}
& {\overline{\left\langle\partial_{x} P(x, t)\right\rangle_{\mathcal{O}_{X_{t}}}}}={\overline{\left\langle\partial_{x} P(x, 0)\right\rangle_{\mathscr{O}_{X_{0}}}}}
\end{aligned}
$$

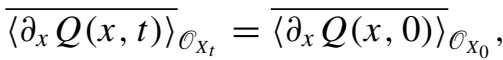

where $\mathscr{O}_{X_{t}}$ is the local ring of the real analytic variety for $X_{t}=F_{t}^{-1}(0)$.

Then the family $F_{t}$ is topologically trivial.

Proof. The proof is analogous to the proof of Theorem 3.1. We use condition $(A)$ and $\left(B_{f}^{*}\right)$ to construct a vector field $V$ which leaves $X=F^{-1}(0)$ invariant.

The next result is an application of the Theorem 3.1 to deformations of Newton non-degenerate map-germs $F_{0}=\left(P_{0}, Q_{0}\right): \mathrm{R}^{n}, 0 \rightarrow \mathrm{R}^{2}, 0$. We first recall the basic notions of Newton diagram of an ideal. 
For this, we fix a coordinate system $x=\left(x_{1}, \ldots, x_{n}\right)$ in $\mathrm{R}^{n}$, so that $\mathscr{A}_{n}$ is identified with the ring $\mathrm{R}[[x]]$ of convergent power series. For each germ $g(x)=\sum a_{k} x^{k}$, we define $\operatorname{supp}(g)=\left\{k \in \mathbf{Z}^{n}: a_{k} \neq 0\right\}$.

DEFINITION 3.5.

(i) Let $I$ be an ideal in $\mathscr{A}_{n}$, define

$$
\operatorname{supp} I=\cup\{\operatorname{supp} g: g \in I\} \text {. }
$$

(ii) The Newton polyhedron of $I$, denoted by $\Gamma_{+}(I)$, is the convex hull in $\mathrm{R}_{+}^{n}$ of the set

$$
\cup\left\{r+v: r \in \operatorname{supp} I, v \in \mathrm{R}_{+}^{n}\right\} .
$$

(iii) $\Gamma(I)$ is the union of all compact faces of $\Gamma_{+}(I)$.

(iv) $I=\left\langle g_{1}, \ldots, g_{s}\right\rangle$ is Newton non-degenerate if for each compact face $\triangle \subset \Gamma(I)$, the equations $g_{1_{\triangle}}(x)=g_{2 \Delta}(x)=\cdots=g_{s \Delta}(x)=0$ have no common solution in $(\mathrm{R} \backslash\{0\})^{n}$, where $g_{i \Delta}$ is the restriction of $g_{i}$ to the face $\triangle$, that is, if $g_{i}(x)=\sum a_{r} x^{r}$ then $g_{i \Delta}(x)=\sum_{r \in \Delta} a_{r} x^{r}$.

Corollary 3.6. Let $F_{0}=\left(P_{0}, Q_{0}\right): \mathrm{R}^{n}, 0 \rightarrow \mathrm{R}^{2}, 0$ and $F(x, t)=$ $\left(P_{0}(x)+t \theta(x), Q_{0}(x)+t \alpha(x)\right)$. Suppose $\Gamma_{+}\left(\partial P_{0}(x)\right)$ and $\Gamma_{+}\left(\partial Q_{0}(x)\right)$ are Newton non-degenerate and $\Gamma_{+}(\partial \theta(x)) \subset \Gamma_{+}\left(\partial P_{0}(x)\right), \Gamma_{+}(\partial \alpha(x)) \quad \subset$ $\Gamma_{+}\left(\partial Q_{0}(x)\right)$.

(1) If $F_{0}$ satisfies condition $(A)$, then $F$ is topologically trivial.

(2) If $F_{0}$ satisfies conditions $(A)$ and $\left(B_{\mathrm{R}}\right)$, then there exists $\epsilon_{0}$ such that for all $0<\epsilon \leq \epsilon_{0}, \frac{F_{t}}{\left\|F_{t}\right\|}: \mathscr{S}_{\epsilon}^{n-1} \backslash K_{t} \rightarrow \mathscr{S}^{1}$ is the projection of a locally trivial fiber bundle, where $K_{t}=F_{t}^{-1}(0) \cap \mathscr{S}_{\epsilon}^{n-1}$. Moreover, $\forall t, t^{\prime} \in \mathrm{R}$, the Milnor fibrations associated to $F_{t}$ and $F_{t^{\prime}}$ are equivalent.

Proof. To verify condition $(A)$ for the family $F_{t}$, let $r: \mathrm{R}, 0 \rightarrow \mathrm{R}^{n}, 0$ be a non constant real analytic curve, with $r(0)=0$.

Therefore, for each $t$, fixed, we have:

$$
\begin{aligned}
\partial_{x} P_{t}(r(s)) & =\partial_{x} P_{0}(r(s))+\partial_{x} R(r(s)) \\
\partial_{x} Q_{t}(r(s)) & =\partial_{x} Q_{0}(r(s))+\partial_{x} S(r(s))
\end{aligned}
$$

Taking the Taylor developments we get,

$$
\begin{aligned}
& \partial_{x} P_{t}(r(s))=\alpha_{1} s^{a_{1}}+\cdots+\alpha_{2} s^{n_{1}}+\cdots \\
& \partial_{x} Q_{t}(r(s))=\beta_{1} s^{a_{1}}+\cdots+\beta_{2} s^{n_{2}}+\cdots .
\end{aligned}
$$

Since $\Gamma_{+}\left(\partial P_{0}(x)\right)$ and $\Gamma_{+}\left(\partial Q_{0}(x)\right)$ are Newton non degenerate, then $a_{1} \leq n_{1}$ and $a_{1} \leq n_{2}$. 
Then,

$$
\begin{gathered}
\frac{\left|\left\langle\partial P_{t}(r(s)), \partial Q_{t}(r(s))\right\rangle\right|}{\left\|\partial P_{t}(r(s))\right\|\left\|\partial Q_{t}(r(s))\right\|}=\frac{\left|\left\langle\alpha_{1} s^{a_{1}}+\cdots, \beta_{1} s^{a_{1}}+\cdots\right\rangle\right|}{\left\|\alpha_{1} s^{a_{1}}+\cdots\right\|\left\|\beta_{1} s^{a_{1}}+\cdots\right\|} \\
=\frac{\left|\left\langle\alpha_{1}, \beta_{1}\right\rangle\right| s^{2 a_{1}}+\cdots}{s^{2 a_{1}}\left\|\alpha_{1}\right\|(1+\cdots)\left\|\beta_{1}\right\|(1+\cdots)}=\frac{\left|\left\langle\alpha_{1}, \beta_{1}\right\rangle\right| s^{2 a_{1}}(1+\cdots)}{\left\|\alpha_{1}\right\|\left\|\beta_{1}\right\| s^{2 a_{1}}(1+\cdots)} \\
=\frac{\left|\left\langle\alpha_{1}, \beta_{1}\right\rangle\right|}{\left\|\alpha_{1}\right\|\left\|\beta_{1}\right\|}+s(\cdots)=\frac{\left|\left\langle\alpha_{1}, \beta_{1}\right\rangle\right|}{\left\|\alpha_{1}\right\|\left\|\beta_{1}\right\|}+u(s)
\end{gathered}
$$

From the hypothesis it follows that $\frac{\left|\left\langle\alpha_{1}, \beta_{1}\right\rangle\right|}{\left\|\alpha_{1}\right\|\left\|\beta_{1}\right\|}<1$ and $\lim _{s \rightarrow 0} u(s)=0$. Then for sufficiently small $s$, we have:

$$
\frac{\left|\left\langle\partial P_{t}(r(s)), \partial Q_{t}(r(s))\right\rangle\right|}{\left\|\partial P_{t}(r(s))\right\|\left\|\partial Q_{t}(r(s))\right\|} \leq 1-\rho
$$

where $0<\rho \leq 1$ for $t \in \mathbf{R}$ fixed. Then, there exist a neighborhood $V \varsubsetneqq \mathbf{R}^{n}$, $0 \in V$ such that

$$
\forall x \in V \backslash\{0\}: \frac{\left|\left\langle\partial P_{t}(x)\right), \partial Q_{t}(x)\right\rangle \mid}{\left\|\partial P_{t}(x)\right\|\left\|\partial Q_{t}(x)\right\|} \leq 1-\rho, \quad 0<\rho \leq 1 .
$$

Since the Newton polyhedrons $\Gamma_{+}\left(\partial P_{0}(x)\right)$ and $\Gamma_{+}\left(\partial Q_{0}(x)\right)$ are non degenerate and $\Gamma_{+}\left(\partial P_{t}(x)\right) \subset \Gamma_{+}\left(\partial P_{0}(x)\right), \Gamma_{+}\left(\partial Q_{t}(x)\right) \subset \Gamma_{+}\left(\partial Q_{0}(x)\right)$, it follows

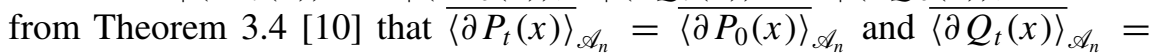

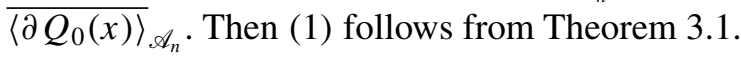

Now, if condition $\left(B_{R}\right)$ holds for $F_{0}$, it follows that $\overline{\left\langle\partial P_{t}(x)\right\rangle_{\mathscr{A}_{n}}}=\overline{\left\langle\partial Q_{t}(x)\right\rangle_{\mathscr{A}_{n}}}$. Thus, we can apply Jacquemard's result to prove that for each $t$, there exists $\epsilon_{0}$, such that for all $0<\epsilon \leq \epsilon_{0}, \frac{F_{t}}{\left\|F_{t}\right\|}: \mathscr{S}_{\epsilon}^{n-1} \backslash K_{t} \rightarrow \mathscr{S}^{1}$ is the projection of a locally trivial fiber bundle. Moreover, it follows from [5] Theorem 1 that these fibrations are equivalent.

ACKNOwLEDGMENTS. I would like to thank my thesis advisor Maria Aparecida Ruas for discussions and numerous remarks on mathematics and English usage of the paper.

\section{REFERENCES}

1. Parusiński, Adam, Topological Triviality of $\mu$-constant deformations of type $f(x)+\operatorname{tg}(x)$, Canad. J. Math. 53 (2001), 73-97.

2. Damon, J., Gaffney, T., Topological triviality of deformations of functions and Newton filtrations, Invent. Math. 72 (1983), 335-358.

3. Gaffney, T., The integral closure of Modules and Whitney equisingularity, Invent. Math. 102 (1992), 301-322. 
4. Martin Greuel, G., Constant Milnor number implies constant multipllicity for quasihomogeneous singularities, Manuscripta Math. 56 (1986), 159-166.

5. King, H. C., Topological type of isolated critical points, Ann. of Math. 107 (1978), 385-397.

6. King, H. C., Topological Type in Families of Germs, Invent. Math. 62 (1980), 1-13.

7. Jaquemard, A., Fibrations de Milnor pour des applications réelles, Boll. Un. Mat. Ital. B (7) 37, 1 (1989), 45-62.

8. Jacquemard, A., Thèse 3ème cycle Université de Dijon, 1982.

9. Milnor, J., Singular Points of Complex Hypersurfaces, Ann. of Math. Stud. 61 (1968).

10. Saia, M. J., The integral closure of ideals and the Newton filtration, J. Algebraic Geom. 6 (1996), 1-11.

11. Ruas, M. A. S., Seade, J., and Verjovsky, On real singularities with a Milnor fibration, Trends Math.: trends in Singularities (2002), 191-213.

12. Teissier, B., Introduction to equisingularity problems, Proc. Sympos. Pure Math. 29 (1975), 539-632.

INSTITUTO DE MATEMÁTICA

UNIVERSIDADE FEDERAL DA BAHIA

AV. ADEMAR DE BARROS, S/N

ONDINA SALVADOR

BAHIA - BRASIL

E-mail: rnonato@ufba.br 